Homology, Homotopy and Applications, vol.22(2), 2020, pp.259-264

\title{
EULER CHARACTERISTICS OF FINITE HOMOTOPY COLIMITS
}

\author{
JOHN D. BERMAN
}

(communicated by Brooke Shipley)

\begin{abstract}
In this note, we provide a calculation of the Euler characteristic of a finite homotopy colimit of finite cell complexes, which depends only on the Euler characteristics of each space and resembles Mobius inversion. Versions of the result are known when the colimit is indexed by categories with various finiteness conditions, but the behavior is more uniform when we index by a finite quasicategory instead. The formula simultaneously generalizes the additive formula for Euler characteristic of a homotopy pushout and the multiplicative formula for Euler characteristic of a fiber bundle.
\end{abstract}

Given a fibration of finite cell complexes $E \rightarrow B$ with $B$ connected and finite fiber $F$, the Euler characteristic satisfies $\chi(E)=\chi(F) \chi(B)$. This is a classical formula with a number of proofs, notably via the Serre Spectral Sequence. In this note, we present a proof (Corollary 1.5) as a special case of a formula for Euler characteristics of homotopy colimits.

We use only the two facts that the Euler characteristic satisfies $\chi(\emptyset)=0$ and $\chi\left(A \cup_{C}^{h} B\right)=\chi(A)+\chi(B)-\chi(C)$, where $\cup^{h}$ denotes a homotopy pushout. Any colimit indexed by a finite category can be built iteratively from the initial object and pushouts. Similarly, any homotopy colimit indexed by a finite $\infty$-category can be built iteratively from the initial object and homotopy pushouts [5, 4.4.2.4]. Therefore, the Euler characteristic of any such homotopy colimit is a linear function of its terms:

Theorem 1.1. If $\mathcal{K}$ is a finite $\infty$-category (see Definition 1.3) and $\mathcal{K} \stackrel{f}{\rightarrow}$ Top is a functor landing in finite $C W$ complexes, then

$$
\chi(\operatorname{colim} f)=\sum_{i \in \pi_{0} \mathcal{K}} \mu(i) \chi(f(i))
$$

where $\pi_{0} \mathcal{K}$ is the set of equivalence classes of objects of $\mathcal{K}$, and $\mu(i)$ are integer constants depending only on $\mathcal{K}$.

Remark 1.2. An $\infty$-category is an object which behaves like a category but is built out of cells like a CW complex. Lurie's Higher Topos Theory [5] is the standard text

The author was supported by an NSF Postdoctoral Fellowship under grant 1803089 . Received September 5, 2019, revised September 19, 2019; published on May 6, 2020. 2010 Mathematics Subject Classification: 55U10, 55M99.

Key words and phrases: Euler characteristic, quasicategory, Mobius function.

Article available at http://dx.doi.org/10.4310/HHA.2020.v22.n2.a16

Copyright (C) 2020, John D. Berman. Permission to copy for private use granted. 
on the subject. Although much of the book is technical, the first chapter is a good introduction.

In this note we explicitly compute the constants $\mu(i)$, which generalize Mobius functions [8] of partially ordered sets, culminating in Theorem 1.7 (at which point we will also provide a more detailed proof of Theorem 1.1).

The idea of such a formula is not new. In fact, the observation that Euler characteristics 'linearize' homotopy colimits is an old one (and a starting point for algebraic K-theory), but the connection to Mobius functions is more recent. Such formulas have been studied by Fiore-Lück-Sauer [1, 2], Leinster [4], and Ponto-Shulman [6, 7], attached to names like the Euler characteristic or Mobius function of categories.

In each case, these authors have to address the question: Which homotopy colimits are linearized? When we pass to $\infty$-categories, the answer becomes straightforward: those indexed by finite $\infty$-categories. The author believes the resulting formula should be of some pedagogical interest to algebraic topologists, as a relatively elementary advertisement of $\infty$-categorical technology.

Definition 1.3. A finite simplicial set is a simplicial set with finitely many nondegenerate simplices. A finite $\infty$-category is an $\infty$-category modeled (in the Joyal model structure on simplicial sets) by a finite simplicial set.

Remark 1.4. Finite $\infty$-categories are very different from finite categories (a fact which may be surprising when first encountered). For example, if $B G$ denotes the category with one object associated to a group $G, B \mathbb{Z} / 2 \cong \mathbb{R} P^{\infty}$ is finite as a category but not as an $\infty$-category, while $B \mathbb{Z} \cong S^{1}$ is finite as an $\infty$-category but not as a category.

Before introducing the main result, we can already prove:

Corollary 1.5. If $F \rightarrow E \rightarrow B$ is a homotopy fiber sequence of finite $C W$ complexes, and $B$ is connected, then $\chi(E)=\chi(F) \chi(B)$.

Lemma 1.6. If $B$ is a finite $C W$ complex, then the associated Kan complex $(\infty-$ groupoid) $\mathcal{B}$, which is unique up to weak equivalence, is finite as an $\infty$-category.

Proof of Lemma 1.6. This proof is taken from an answer of Simon Henry on Mathoverflow [3]. Suppose $X$ is a finite CW complex, modeled (in the classical Quillen model structure on simplicial sets) as a finite simplicial set. Label the nondegenerate 1 -simplices of $X$ as $e_{1}, \ldots, e_{n}$. For each $1 \leqslant i \leqslant n$, add two 2 -simplices $c_{i}, c_{i}^{\prime}$ to $X$ with faces $s_{0} c_{i}=s_{2} c_{i}^{\prime}=e_{i}, s_{1} c_{i}=s_{1} c_{i}^{\prime}=\mathrm{id}$. That is, we add both a left inverse $s_{2} c_{i}$ and a right inverse $s_{0} c_{i}^{\prime}$ to $e_{i}$. In particular, we do this via iterated pushouts along horns $X \amalg_{\Lambda^{0}[2]} \Delta[2]$ and $X \amalg_{\Lambda^{2}[2]} \Delta[2]$.

The new simplicial set $X^{\prime}$ is also finite, and the inclusion $X \rightarrow X^{\prime}$ is a weak equivalence because each pushout did not change the homotopy type of $X$. (That is, $X^{\prime}$ still models $X$ as a finite CW complex.) Moreover, since every 1-simplex in $X^{\prime}$ has either a right or left inverse, $X^{\prime}$ models an $\infty$-groupoid in the Joyal model structure, which is necessarily the space $X$. Therefore $X$ is finite as an $\infty$-groupoid. 
Proof of Corollary 1.5. Let $\mathcal{B}$ denote the $\infty$-groupoid associated to $B$, whose objects are points of $B$. There is a functor $E_{-}: \mathcal{B} \rightarrow$ Top which sends a point $b \in B$ to the homotopy fiber $E_{b}=E \times{ }_{B}^{h} *$, and the total space $E$ can be reconstructed as the colimit $[\mathbf{5}, 3.3 .4 .6]$

$$
E \cong \operatorname{colim} E_{-} .
$$

Since $\mathcal{B}$ is finite and $\left|\pi_{0} \mathcal{B}\right|=1$, Theorem 1.1 asserts

$$
\chi(E)=\mu(*) \chi\left(E_{*}\right)=\mu_{B} \chi(F),
$$

where $*$ is the single element of $\pi_{0} \mathcal{B}$ and $\mu_{B}$ is a constant that depends only on $B$.

To compute $\mu_{B}$, consider the fiber sequence $* \rightarrow B \rightarrow B$. We have $\chi(B)=\mu_{B} \chi(*)$, so $\mu_{B}=\chi(B)$. This completes the proof.

We will now extend Theorem 1.1 by explicitly computing the constants $\mu(i)$. Theorem 1.7 categorifies the usual formula for $\chi(X)$ given a simplicial model for the space $X$. (See Example 1.11.)

Theorem 1.7. If $K$ is a finite simplicial set, and $x \in K_{0}$ is an object (0-simplex), the Mobius function is $\mu(x)=\sum(-1)^{n}\left|K_{n}^{x}\right|$. Here $\left|K_{n}^{x}\right|$ denotes the number of nondegenerate $n$-simplices with initial vertex $x$.

If $K \stackrel{f}{\rightarrow}$ Top is a functor that lands in finite cell complexes,

$$
\chi(\operatorname{colim} f)=\sum_{x \in K_{0}} \mu(x) \chi(f(x)) .
$$

Remark 1.8. The connection between Theorems 1.1 and 1.7 is as follows: If $K$ is a finite simplicial set model for the $\infty$-category $\mathcal{K}$ and $i \in \pi_{0} \mathcal{K}$ is an equivalence class of objects, then

$$
\mu_{\mathcal{K}}(i)=\sum_{x \cong i} \mu_{K}(x)
$$

Proof of Theorems 1.1 and 1.7. The proof exactly follows Lurie's proof that all finite colimits (of $\infty$-categories) can be built out of pushouts and initial objects $[\mathbf{5}, 4.4 .2 .4]$. We take for granted that the Euler characteristic is 0 on the empty space and takes homotopy pushouts to sums (as in Example 1.12).

Choose $K$ finite and $K \stackrel{f}{\rightarrow}$ Top which lands in finite cell complexes. We induct first on the dimension of $K$, then on the number of simplices of maximal dimension. When $K$ is 0 -dimensional, the theorems hold by $\chi(X \amalg Y)=\chi(X)+\chi(Y)$.

Otherwise, fix a nondegenerate simplex $\Delta^{n}$ in $K$ of maximal dimension, with initial vertex $s$ and terminal vertex $t$. Decompose $K \cong K^{\prime} \amalg_{\partial \Delta^{n}} \Delta^{n}$, and let $\mu$ be the Mobius function on $K, \mu^{\prime}$ the Mobius function on $K^{\prime}$ (as defined in Theorem 1.7). Note that $K$ has the same objects as $K^{\prime}$ since $n \geqslant 1$. Moreover, $\mu(x)=\mu^{\prime}(x)$, except at $x=s$, where $\mu(s)=\mu^{\prime}(s)+(-1)^{n}$. 
Now we compute the colimit. Let

$$
\begin{gathered}
X=\operatorname{colim}\left(\left.f\right|_{K^{\prime}}\right), \\
Y=\operatorname{colim}\left(\left.f\right|_{\partial \Delta^{n}}\right), \\
Z=\operatorname{colim}\left(\left.f\right|_{\Delta^{n}}\right) .
\end{gathered}
$$

Then $\operatorname{colim}(f)=X \amalg_{Y} Z$, so $\chi(\operatorname{colim} f)=\chi(X)+\chi(Z)-\chi(Y)$. By the induction hypothesis, $\chi(Y)=\chi(f(t))-(-1)^{n} \chi(f(s))$ and $\chi(Z)=\chi(f(t))$, so

$$
\chi(\operatorname{colim} f)=\chi(X)+(-1)^{n} \chi(f(s))=\sum_{x} \mu^{\prime}(x) \chi(f(x))+(-1)^{n} \chi(f(s)) .
$$

Since $\mu(x)=\mu^{\prime}(x)$ and $\mu(s)=\mu^{\prime}(s)+(-1)^{n}$, this completes the proof. Theorem 1.1 follows as described by Remark 1.4.

Remark 1.9. Notice that we have proven something slightly more general. Suppose $\mathcal{C}$ is an $\infty$-category which admits finite colimits, $A$ is an abelian group, and $\chi: \pi_{0} \mathcal{C} \rightarrow A$ is a function satisfying the two conditions:

- if 0 is the initial object, then $\chi(0)=0$;

- if

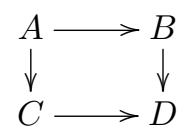

is a homotopy pushout square, then $\chi(A)+\chi(D)=\chi(B)+\chi(C)$.

Then for any finite $\infty$-category $\mathcal{K}$ and functor $\mathcal{K} \stackrel{f}{\rightarrow} \mathcal{C}$, we have

$$
\chi(\operatorname{colim} f)=\sum_{i \in \pi_{0} \mathcal{K}} \mu(i) \chi(f(i))
$$

We will end this note with some examples.

Notice that the Mobius function of Theorem 1.7 recovers the classical Mobius function [8] of a finite poset. Let $P$ be a finite poset with upper bound 1 (so $x \leqslant 1$ for all $x)$. Let $\mu_{P}: P \rightarrow \mathbb{Z}$ be the classical Mobius function. That is, if $g, h: P \rightarrow \mathbb{R}$ satisfy

$$
h(y)=\sum_{x \leqslant y} g(x)
$$

then

$$
g(1)=\sum_{x \in P} \mu_{P}(x) h(x)
$$

Let $P_{0}=P-\{1\}$, also a finite poset, and note that any (finite) poset is canonically a (finite) $\infty$-category, via the nerve of the associated category.

If $\mu_{0}$ denotes our Mobius function (a la Theorem 1.7), then $\mu_{0}(x)=-\mu_{P}(x)$ for any $x \in P_{0}$, while $\mu_{P}(1)=1$. Hence, we can restate Theorem 1.7 in terms of the classical Mobius function: If $P \stackrel{f}{\rightarrow}$ Top is a functor landing in finite cell complexes 
which exhibits $f(1)$ as a colimit of $\left.f\right|_{P_{0}}$, then

$$
0=\chi(f(x))-\chi\left(\left.\operatorname{colim} f\right|_{P_{0}}\right)=\sum_{x \in P} \mu_{P}(x) \chi(f(x)) .
$$

Applying classical Mobius inversion:

Corollary 1.10 (Mobius inversion). If $P$ is a finite poset, $P \stackrel{f}{\rightarrow}$ Top a functor for which each $f(x)$ is a finite cell complex, $\chi f(x)$ the Euler characteristic of $f(x)$, and $d \chi f(x)=\chi(f(x))-\chi\left(\left.\operatorname{colim} f\right|_{<x}\right)$ the Euler characteristic obstruction to $f(x)$ being the colimit of $f: P_{<x} \rightarrow$ Top, then

$$
\chi f(y)=\sum_{x \leqslant y} d \chi f(x) .
$$

Many classical properties of Euler characteristics are special cases of Theorems 1.1 and 1.7:

Example 1.11 ( $\chi$ of a simplicial complex). For a finite simplicial set (or finite $\infty$ category) $K$, the classifying space $|K|$ is the colimit of the constant diagram at a point $K \stackrel{*}{\rightarrow}$ Top $[\mathbf{5}, 4.4 .4 .9]$. Therefore, the Euler characteristic of its classifying space is given by

$$
\chi|K|=\sum_{x \in K_{0}} \mu(x)=\sum_{n \geqslant 0}(-1)^{n}\left|K_{n}\right| .
$$

We recover the classical fact that the Euler characteristic of a simplicial complex is the alternating sum of the numbers of $n$-simplices.

Example 1.12 ( $\chi$ of a homotopy pushout). Given a homotopy pushout

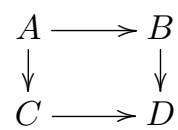

of finite cell complexes, then $\chi(D)=\chi(B)+\chi(C)-\chi(A)$.

Example 1.13 ( $\chi$ of a fiber bundle). Regard a finite CW complex $B$ as an $\infty$-groupoid, also finite by Lemma 1.6. If $B$ is connected and $F \rightarrow E \rightarrow B$ is a homotopy fiber sequence of finite $\mathrm{CW}$ complexes, then

$$
\chi(E)=\chi\left(\operatorname{colim} E_{-}\right)=\chi(F) \chi(B) .
$$

This is precisely Corollary 1.5 .

\section{References}

[1] T. Fiore, W. Lück, and R. Sauer. Euler characteristics of categories and homotopy colimits. Documenta Mathematics, 16:301-354 (2011).

[2] T. Fiore, W. Lück, and R. Sauer. Finiteness obstructions and Euler characteristics of categories. Advances in Mathematics, 226:2371-2469 (2011).

[3] S. Henry. Why are finite cell complexes also finite as infinity-categories? URL (version 2018-12-6): https://mathoverflow.net/questions/314690/why-are-finitecell-complexes-also-finite-as-infinity-categories. 
[4] T. Leinster. The Euler characteristic of a category. Documenta Mathematica, 13:21-49 (2008).

[5] J. Lurie. Higher Topos Theory. Annals of Mathematics Studies, 170, Princeton University Press. Princeton, NJ (2009).

[6] K. Ponto and M. Shulman. The linearity of traces in monoidal categories and bicategories. Theory and Applications of Categories, 31:594-689 (2016).

[7] K. Ponto and M. Shulman. The multiplicativity of fixed point invariants. Algebraic and Geometric Topology, 14:1275-1306 (2014).

[8] G. Rota. On the foundations of combinatorial theory I: theory of Möbius functions. Zeitschrift für Wahrscheinlichkeitstheorie und Verwandte Gebiete, $2(4): 340-368$ (1964).

John D. Berman jberman@math.utexas.edu

Department of Mathematics, The University of Texas at Austin, 2515 Speedway, RLM 8.100, Austin, TX 78712, USA 